\title{
GATA6 regulates epithelial-mesenchymal transition and metastasis through regulating MUC1/ $\beta$-catenin pathway in cholangiocarcinoma
}

\section{Xiang Deng}

Southwest Hospital

\section{Peng Jiang}

Southwest Hospital

Jian Chen

Southwest Hospital

Jianwei Li

Southwest Hospital

Dajiang Li

Southwest Hospital

Yu He

Southwest Hospital

Yan Jiang

Southwest Hospital

Yujun Zhang

Southwest Hospital

Shuangnian $\mathrm{Xu}$

Southwest Hospital

Xi Li

Southwest Hospital

Shuguang Wang

Southwest Hospital

Feng Tian ( $\square$ tianfeng@tmmu.edu.cn )

Third Military Medical University Southwest Hospital https://orcid.org/0000-0002-1964-4661

\section{Keywords:}

Posted Date: July 28th, 2020

DOI: https://doi.org/10.21203/rs.3.rs-20809/v2 
License: (c) (i) This work is licensed under a Creative Commons Attribution 4.0 International License. Read Full License 


\section{Abstract}

The authors have requested that this preprint be removed from Research Square. 